\title{
Extended supersymmetric sigma-models in 3D AdS
}

\author{
Gabriele Tartaglino-Mazzucchelli*t \\ School of Physics M013, The University of Western Australia, \\ 35 Stirling Highway, Crawley W.A. 6009, Australia \\ E-mail: gabriele.tartaglino-mazzucchelli@uwa.edu.au
}

We review our recent construction of three-dimensional (3D) $(p, q)$ Anti-de Sitter superspaces and the classification of the target space geometries associated with general supersymmetric $\mathscr{N}=$ 2,3,4 nonlinear sigma-models in $\mathrm{AdS}_{3}$.

Proceedings of the Corfu Summer Institute 2012 "School and Workshops on Elementary Particle Physics and Gravity"

September 8-27, 2012

Corfu, Greece

\footnotetext{
* Speaker.

†We thank the organizers of the Corfu Summer Institute 2012 "School and Workshops on Elementary Particle Physics and Gravity" for the opportunity to present our work.
} 


\section{Introduction}

The last few years have seen a renewed interest in 3D supersymmetric theories. On the one hand this was generated by the research triggered by the study of M2-brane dynamics and 3D superconformal Chern-Simons theories [1,2,3]. On the other hand, new insights into the dynamics of 3D massive gravity theories have been achieved. In particular Topological-Massive-Gravity (TMG) [4], New-Massive-Gravity (NMG) and Generalized-Massive-Gravity (GMG), [5] received a lot of attention. All these models include three-dimensional $\mathrm{AdS}_{3}$ space-time as a maximally symmetric solution allowing for extended supersymmetry.

Superspace techniques can be used to construct general $\mathscr{N}$-extended supergravity models in 3D. The $\mathscr{N}=1$ case was studied in [6,7]. The $\mathscr{N} \geq 2$ cases were sketched in [8,9] and then developed, in collaboration with S. M. Kuzenko and U. Lindström, in [10] for $\mathscr{N}$-extended conformal supergravity. The advantage of the superspace approach is that, extending the superconformal results of [11], we provided formalisms to study general supergravity-matter systems with $\mathscr{N} \leq 4$.

By using components techniques, long ago, Achúcarro and Townsend gave a classification of 3D $\mathscr{N}$-extended anti-de Sitter (AdS) supergravity called $(p, q)$ supergravities [12]. The two non-negative integers $p \geq q$ are such that $\mathscr{N}=p+q$ and they classify the in-equivalent isometry supergroups $\operatorname{OSp}(\mathrm{p} \mid 2 ; \mathbb{R}) \times \operatorname{OSp}(\mathrm{q} \mid 2 ; \mathbb{R})$ of the AdS backgrounds.

In [13], using the results of [10], we deepened the study of 3D $\mathscr{N}=2$ AdS supergravities in superspace and their $(1,1)$ and $(2,0)$ AdS geometry. Furthermore, we elaborated on rigid supersymmetric theories in $(1,1)$ and $(2,0)$ AdS superspaces. The advantage in our superspace construction was the possibility to write general matter couplings in the AdS supergravities.

Recently there was a renewed interest in studying field theories in curved spaces (see [14, 15, $16,17,18])$. New superspace techniques for rigid supersymmetric theories on curved background have clear applications if one is interested to lift off-shell theories from flat to curved backgrounds [18]. Such problems have recently arisen in studying the partition function of gauge theories on nontrivial, constant-curvature backgrounds when computing observables such as expectation values of Wilson loops and superconformal indices by using localization techniques [19].

A natural question was to complete the classification of AdS supersymmetry in superspace. The classification of 3D $(p, q)$ AdS superspaces and the study of general $\mathscr{N}=3,4$ supersymmetric nonlinear sigma-models was given in [20, 21]. Compared to supersymmetry in 3D Minkowski, in which the $\mathscr{N}=3,4$ sigma-model target spaces is a general hyper-Kähler manifold [11], the various AdSs impose further non-trivial constraints. The target spaces of $\mathscr{N}=3,4$ AdS sigmamodels prove to be typically: (i) hyper-Kähler cones; and (ii) hyper-Kähler spaces with a U(1) isometry group which acts non-trivially on the two-sphere of complex structures. In some critical, $\mathscr{N}=4$, cases it is even possible to have unconstrained hyper-Kähler spaces.

This report is devoted to a review of the results of $[10,13,20,21]$ with an ultimate focus on the results for the target space geometries of $\mathscr{N}=2,3,4$ supersymmetric non-linear sigma-models in $\mathrm{AdS}_{3}$. Our review is organized as follow: In section 2, we review the superspace formulation of 3D $(p, q)$ AdS superspaces as a background of $\mathscr{N}$-extended conformal supergravity. In section 3, we describe the properties of supersymmetric sigma-moldels in $\mathscr{N}=2$ AdS. Section 4 is devoted to describe the target space geometries of the $\mathscr{N}=3,4$ supersymmetric sigma-models. In section 5 we finish with few conclusive comments. 


\section{Three-dimensional $(p, q)$ AdS superspaces}

Our analysis of the sigma-model target space geometries will be based on the use of superspace techniques. In this section, we then review the main properties of the differential geometry of threedimensional $\mathscr{N}$-extended $(p, q)$ AdS superspaces. We start by rewiewing the superspace geometry of conformal supergravity.

\subsection{Superspace geometry of $\mathscr{N}$-extended conformal supergravity}

All $(p, q)$ AdS superspaces can be realised as special background configurations within the 3D $\mathscr{N}$-extended conformal supergravity geometry that was originally sketched in [8] and then developed in [10].

In three dimensions, $\mathscr{N}$-extended conformal supergravity can be described by using a curved superspace which is parametrized by real bosonic $\left(x^{m}\right)$ and real fermionic $\left(\theta_{I}^{\mu}\right)$ coordinates,

$$
z^{M}=\left(x^{m}, \theta_{I}^{\mu}\right), \quad m=0,1,2, \quad \mu=1,2, \quad I=1, \cdots, \mathscr{N},
$$

and is characterised by the structure group $\operatorname{SL}(2, \mathbb{R}) \times \mathrm{SO}(\mathscr{N})$. The superspace differential geometry is encoded in covariant derivatives of the form

$$
\mathscr{D}_{A} \equiv\left(\mathscr{D}_{a}, \mathscr{D}_{\alpha}^{I}\right)=E_{A}{ }^{M} \partial_{M}+\frac{1}{2} \Omega_{A}{ }^{c d} \mathscr{M}_{c d}+\frac{1}{2} \Phi_{A}{ }^{K L} \mathscr{N}_{K L},
$$

where $E_{A}=E_{A}{ }^{M} \partial_{M}$ is the supervielbein, with $\partial_{M}=\partial / \partial z^{M} ; \mathscr{M}_{b c}$ and $\Omega_{A}{ }^{b c}$ are respectively the Lorentz generators and connections; $\mathscr{N}_{K L}$ and $\Phi_{A}{ }^{K L}$ are respectively the $\operatorname{SO}(\mathscr{N})$ generators and connections. For more details on our notation and conventions see Appendix A of [10].

To describe conformal supergravity, one can impose a set of conventional constrains [8]. Then the Bianchi identities, $\sum_{[A B C)}\left[\left[\mathscr{D}_{A}, \mathscr{D}_{B}\right\}, \mathscr{D}_{C}\right\}=0$, lead to the (anti) commutation relations ${ }^{1}[10]$ :

$$
\begin{aligned}
\left\{\mathscr{D}_{\alpha}^{I}, \mathscr{D}_{\beta}^{J}\right\}= & 2 \mathrm{i} \delta^{I J} \mathscr{D}_{\alpha \beta}-2 \mathrm{i} \varepsilon_{\alpha \beta} C^{\gamma \delta I J} \mathscr{M}_{\gamma \delta}-4 \mathrm{i} S^{I J} \mathscr{M}_{\alpha \beta} \\
& +\left(\mathrm{i} \varepsilon_{\alpha \beta} X^{I J K L}-4 \mathrm{i} \varepsilon_{\alpha \beta} S^{K I} \delta^{J] L}+\mathrm{i} C_{\alpha \beta}{ }^{K L} \delta^{I J}-4 \mathrm{i} C_{\alpha \beta}{ }^{K(I} \delta^{J) L}\right) \mathscr{N}_{K L} \\
{\left[\mathscr{D}_{\alpha \beta}, \mathscr{D}_{\gamma}^{K}\right]=} & -\left(\varepsilon_{\gamma(\alpha} C_{\beta) \delta}{ }^{K L}+\varepsilon_{\delta(\alpha} C_{\beta) \gamma}{ }^{K L}+2 \varepsilon_{\gamma(\alpha} \varepsilon_{\beta) \delta} S^{K L}\right) \mathscr{D}_{L}^{\delta}+\cdots
\end{aligned}
$$

where the ellipses indicate dimension-3/2 curvature terms. The algebra is parametrized by three tensor superfields of mass dimension- $1, X^{I J K L}, S^{I J}$ and $C_{a}{ }^{I J}$, which are real and have the symmetry properties: $X^{I J K L}=X^{[I J K L}, S^{I J}=S^{(I J)}$ and $C_{a}{ }^{I J}=C_{a}{ }^{[I J]}$. The components of the curvatures of higher mass dimension can be determined by solving the Bianchi identities and are function of the dimension- 1 tensor superfields and their covariant derivatives. The Bianchi identities also imply a set of differential constraints on the various dimension-1 fields as described in [10].

The supergravity gauge group is generated by local transformations that act on the covariant derivatives $\mathscr{D}_{A}$ and on a tensor superfield $T$ respectively as

$$
\delta_{K} \mathscr{D}_{A}=\left[K, \mathscr{D}_{A}\right], \quad \delta_{K} T=K T, \quad K=K^{C} \mathscr{D}_{C}+\frac{1}{2} K^{c d} \mathscr{M}_{c d}+\frac{1}{2} K^{P Q} \mathscr{N}_{P Q}
$$

\footnotetext{
${ }^{1}$ We only give the explicit expressions for the mass dimension- 1 components of the torsion and the curvatures.
} 
The gauge parameters $K^{C}, K^{c d}$ and $K^{P Q}$ obey natural reality conditions but are otherwise arbitrary.

The fact that the previous superspace geometry describes conformal supergravity relies on its invariance under the following super-Weyl transformations ${ }^{2}$

$$
\begin{aligned}
\delta_{\sigma} \mathscr{D}_{\alpha}^{I} & =\frac{1}{2} \sigma \mathscr{D}_{\alpha}^{I}+\left(\mathscr{D}^{\beta I} \sigma\right) \mathscr{M}_{\alpha \beta}+\left(\mathscr{D}_{\alpha J} \sigma\right) \mathscr{N}^{I J}, \\
\delta_{\sigma} \mathscr{D}_{a} & =\sigma \mathscr{D}_{a}+\frac{\mathrm{i}}{2}\left(\gamma_{a}\right)^{\alpha \beta}\left(\mathscr{D}_{\alpha}^{K} \sigma\right) \mathscr{D}_{\beta K}+\varepsilon_{a b c}\left(\mathscr{D}^{b} \sigma\right) \mathscr{M}^{c}+\frac{\mathrm{i}}{16}\left(\gamma_{a}\right)^{\alpha \beta}\left(\left[\mathscr{D}_{\alpha}^{[K}, \mathscr{D}_{\beta}^{L]}\right] \sigma\right) \mathscr{N}_{K L}, \\
\delta_{\sigma} S^{I J} & =\sigma S^{I J}-\frac{\mathrm{i}}{8}\left(\left[\mathscr{D}^{\gamma(I}, \mathscr{D}_{\gamma}^{J)}\right] \sigma\right), \\
\delta_{\sigma} C_{a}^{I J} & =\sigma C_{a}^{I J}-\frac{\mathrm{i}}{8}\left(\gamma_{a}\right)^{\alpha \beta}\left(\left[\mathscr{D}_{\alpha}^{[I}, \mathscr{D}_{\beta}^{J]}\right] \sigma\right), \\
\delta_{\sigma} X^{I J K L} & =\sigma X^{I J K L} .
\end{aligned}
$$

These provide a non-linear realization of local dilatations, $S$-supersymmetry and conformal boosts transformations whose transformation parameters are all encoded in the real unconstrained superfield $\sigma$. Note that the superfield $X^{I J K L}$ transforms homogeneously and is a superspace generalization of the 3D Cotton tensor. Various components of the supergravity multiplet can be algebraically gauged away leaving as physical fields: the vielbein $e_{a}{ }^{m}$; the gravitino $\Psi_{a}{ }^{\mu}$, the $\mathrm{SO}(\mathscr{N})$ connection $A_{a}{ }^{K L}$; a tower of auxiliary fields whose lowest mass dimensional component is $\left.X^{I J K L}\right|_{\theta=0}$.

\subsection{Definition of $(p, q)$ AdS superspaces}

We define the $(p, q)$ AdS superspaces to be those conformal supergravity backgrounds which satisfy the following requirements [20]: (i) the torsion and curvature tensors are Lorentz invariant; (ii) the torsion and curvature tensors are covariantly constant. These conditions imply

$$
C_{a}^{I J} \equiv 0, \quad \mathscr{D}_{\alpha}^{I} S^{J K}=\mathscr{D}_{a} S^{J K}=0, \quad \mathscr{D}_{\alpha}^{I} X^{J K L M}=\mathscr{D}_{a} X^{J K L M}=0 .
$$

The complete algebra of covariant derivatives then takes the form:

$$
\begin{aligned}
\left\{\mathscr{D}_{\alpha}^{I}, \mathscr{D}_{\beta}^{J}\right\} & =2 \mathrm{i} \delta^{I J} \mathscr{D}_{\alpha \beta}-4 \mathrm{i} S^{I J} \mathscr{M}_{\alpha \beta}+\mathrm{i} \varepsilon_{\alpha \beta}\left(X^{I J K L}-4 S^{K[I} \delta^{J] L}\right) \mathscr{N}_{K L}, \\
{\left[\mathscr{D}_{a}, \mathscr{D}_{\beta}^{J}\right] } & =S^{J}{ }_{K}\left(\gamma_{a}\right)_{\beta}{ }^{\gamma} \mathscr{D}_{\gamma}^{K}, \\
{\left[\mathscr{D}_{a}, \mathscr{D}_{b}\right] } & =-4 S^{2} \mathscr{M}_{a b}, \quad S^{2}:=\frac{1}{\mathscr{N}} S^{I J} S_{I J} \geq 0 .
\end{aligned}
$$

Note that, when $S>0$, the vector-vector commutator in (2.7c) is exactly that of $\mathrm{AdS}_{3}$ with constant curvature parameter $S$. When $S=0$ the bosonic body of the superspace is flat.

The previous algebra clearly needs to satisfy the Bianchi identities. Moreover, for consistency of the condition (ii), the superfields $S^{I J}$ and $X^{I J K L}$ have to satisfy the integrability conditions

$$
\left\{\mathscr{D}_{\alpha}^{I}, \mathscr{D}_{\beta}^{J}\right\} S^{K L}=0, \quad\left\{\mathscr{D}_{\alpha}^{I}, \mathscr{D}_{\beta}^{J}\right\} X^{K L M N}=0 .
$$

By analyzing the Bianchi identities and eq. (2.8) one gets the following constraint on $S^{I J}$ :

$$
S^{I K} S_{K}^{J}=S^{2} \delta^{I J} \text {. }
$$

\footnotetext{
${ }^{2}$ In the general $\mathscr{N}$-extended conformal supergravity case, the super-Weyl transformations were described in full details in [10, 20]. For $\mathscr{N}=8$ supergravity, the finite form of super-Weyl transformations first appeared in [9].
} 
When $S^{2}>0$, which correspond to a non-trivial $\mathrm{AdS}_{3}$ background, $S^{I J}$ is a nonsingular symmetric $\mathscr{N} \times \mathscr{N}$ matrix, and, due to (2.9), $S^{I J} / S$ is an orthogonal matrix. Local $\mathrm{SO}(\mathscr{N})$ transformations can be then used to diagonalise $S^{I J}$ in the form

$$
S^{I J}=S \operatorname{diag}(\overbrace{+1, \cdots,+1}^{p}, \overbrace{-1, \cdots,-1}^{q=\mathscr{N}-p}), \quad S>0 .
$$

Such a frame is called diagonal frame. In this frame the structure group of the AdS superspace is reduced from $\mathrm{SO}(\mathscr{N})$ to $\mathrm{SO}(p) \times \mathrm{SO}(q)$. This corresponds to the $R$-symmetry group of the classification of $(p, q)$ supersymmetry in $\mathrm{AdS}_{3}$ originally introduced in [12].

Given $S^{I J}$ of a particular $(p, q)$ AdS superspace, we can then analyze the implications of the Bianchi identities and of the integrability condition (2.8) on the curvature superfield $X^{I J K L}$. There are two different cases:

$$
\begin{aligned}
& q>0: \quad \Longrightarrow \quad X^{I J K L}=0, \\
& (\mathscr{N}, 0): \quad \Longrightarrow \quad X_{N}{ }^{I J[K} X^{L P Q] N}=0 .
\end{aligned}
$$

These equations tell us that the covariantly constant superfield $X^{I J K L}$ act as a deformation parameter for $(\mathscr{N}, 0)$ superspaces, while for $(p, q)$ AdS superspaces with $q>0$ such deformation is not allowed. When $\mathscr{N}=1,2,3$ the superfield $X^{I J K L}$, being completely antisymmetric, does not exist and $(2.11 \mathrm{~b})$ can be neglected. On the other hand, it is of particular interest the first non-trivial case, $\mathscr{N}=4$, where the general solution of $(2.11 b)$ is given by:

$$
\mathscr{N}=4: \quad X^{I J K L}=\varepsilon^{I J K L} X,
$$

with $\varepsilon^{I J K L}$ the $\mathrm{SO}(4)$ invariant Levi-Civita tensor $\left(\varepsilon^{1234}=1\right)$ and $X$ an arbitrary real scalar and $\mathrm{SO}(4)$ invariant superfield [20]. Such a deformation is possible thanks to the violation of the Dragon's theorem in 3D [10]. In dimensions higher than three similar deformations are not known.

It is interesting to notice that when $S=0$ the consistent algebra of covariant derivatives is

$$
\begin{aligned}
\left\{\mathscr{D}_{\alpha}^{I}, \mathscr{D}_{\beta}^{J}\right\} & =2 \mathrm{i} \delta^{I J} \mathscr{D}_{\alpha \beta}+\mathrm{i} \varepsilon_{\alpha \beta} X^{I J K L} \mathscr{N}_{K L}, \\
{\left[\mathscr{D}_{a}, \mathscr{D}_{\beta}^{J}\right] } & =0, \quad\left[\mathscr{D}_{a}, \mathscr{D}_{b}\right]=0 .
\end{aligned}
$$

This superspace is of Minkowski type for $\mathscr{N}=1,2,3$. However, for $\mathscr{N} \geq 4$ there may exist a non-zero constant antisymmetric tensor $X^{I J K L}=\varepsilon^{I J K L} X$ such that the resulting superspace is a deformation of the $\mathscr{N}$-extended Minkowski superspace.

\subsection{Maximal supersymmetry and conformal flatness of $(p, q)$ AdS superspaces}

One of the main properties of the AdS backgrounds in various dimension is that the supersymmetry transformation preserve the same number of supersymmetry as in the flat case. By using our superspace construction, it is indeed possible to see that every $(p, q)$ AdS superspace preserve all the $\mathscr{N}$ supersymmetries. To investigate this statement, we can proceed by studying the isometry transformations in a given $(p, q)$ superspace. These, by definition, are a particular combination of diffeomorphism and structure group transformations that preserve the covariant derivatives. In particular, they are generated by Killing vector fields

$$
\xi=\xi^{a} \mathscr{D}_{a}+\xi_{I}^{\alpha} \mathscr{D}_{\alpha}^{I}
$$


which by definition obeys the equation

$$
\left[\xi+\frac{1}{2} \Lambda^{I J} \mathscr{N}_{I J}+\frac{1}{2} \Lambda^{a b} \mathscr{M}_{a b}, \mathscr{D}_{C}\right]=0,
$$

for some parameters $\Lambda^{I J}$ and $\Lambda^{a b}$. It turns out that the previous equation completely define $\xi_{I}^{\alpha}, \Lambda^{I J}$ and $\Lambda^{a b}$ in terms of first spinor derivatives of $\xi^{a}$ [10]. The latter is then subject to extra constraints including the standard Killing vector equation

$$
\mathscr{D}_{a} \xi_{b}+\mathscr{D}_{b} \xi_{a}=0 .
$$

In this description of the isometry group, the supersymmetry transformation parameters, are the $\theta$-independent component of $\xi_{I}^{\alpha}: \varepsilon_{I}^{\alpha}:=\left.\xi_{I}^{\alpha}\right|_{\theta=0}$. The number of real independent supersymmetry parameters is $2 \mathscr{N}=2(p+q)$ exactly as in the flat limit. Hence the $(p, q) \operatorname{AdS}_{3}$ backgrounds are maximally supersymmetric. Note that in the diagonal frame $\Lambda^{I J}$ takes value in $\mathrm{SO}(p) \times \mathrm{SO}(q)$. Indeed, it turns out that, with $X^{I J K L}=0$, the full isometry group is factorized and the Killing vector fields parametrize the $\operatorname{AdS}$ supergroup $\operatorname{OSp}(p \mid 2 ; \mathbb{R}) \times \operatorname{OSp}(q \mid 2 ; \mathbb{R})$. For the $(\mathscr{N}, 0)$ and $X^{I J K L} \neq 0$ cases, the isometry group is deformed and there are cases in which the $R$-symmetry group is a proper subgroup of $\mathrm{SO}(\mathscr{N})$. We will give specific examples in the $\mathscr{N}=4$ case.

It is well known that in any dimensions the AdS space-times are conformally flat. In four dimensions it was proven that all $\mathscr{N}$-extended AdS superspaces are conformally flat [22]. A natural question is wether our $(p, q)$ AdS superspaces are conformally flat. Because the $X^{I J K L}$ superfield in the conformal supergravity geometry represent the supersymmetric extension of the Cotton tensor, it can be proven that a necessary and sufficient condition for a background to be conformally flat is that $X^{I J K L}=0$. This implies that our $(p, q)$ AdS superspaces are conformally flat iff the deformation parameter $X^{I J K L}=0$. In this case, it is possible to explicitly construct a local conformally flat parametrization of the $(p, q)$ AdS superspaces. This is defined in terms of a superfield $\sigma$ such that

$$
\begin{aligned}
& \mathrm{e}^{\sigma}=1-s^{2} x^{2}-\mathrm{i} \Theta_{s}-\frac{1}{8} s^{2}(\Theta)^{2}, \quad \theta^{I J}:=\theta^{\gamma I} \theta_{\gamma}^{J}=\theta^{J I}, \\
& s:=\sqrt{s^{K L} S_{K L} / \mathscr{N}}=S, \quad s^{I J}=\mathrm{const}, \quad \Theta_{s}:=s^{I J} \theta_{I J}, \Theta:=\delta^{I J} \theta_{I J} .
\end{aligned}
$$

The covariant derivatives and the covariantly constant $S^{I J}$ tensor take the form

$$
\begin{aligned}
\mathscr{D}_{\alpha}^{I}= & \mathrm{e}^{\frac{1}{2} \sigma}\left(D_{\alpha}^{I}+\left(D^{\beta I} \sigma\right) \mathscr{M}_{\alpha \beta}+\left(D_{\alpha J} \sigma\right) \mathscr{N}^{I J}\right) \\
\mathscr{D}_{a}= & \mathrm{e}^{\sigma}\left(\partial_{a}+\frac{\mathrm{i}}{2}\left(\gamma_{a}\right)^{\alpha \beta}\left(D_{(\alpha}^{K} \sigma\right) D_{\beta) K}+\varepsilon_{a b c}\left(\partial^{b} \sigma\right) \mathscr{M}^{c}-\frac{\mathrm{i}}{8}\left(\gamma_{a}\right)^{\alpha \beta}\left(D_{K}^{\rho} \sigma\right)\left(D_{\rho}^{K} \sigma\right) \mathscr{M}_{\alpha \beta}\right. \\
& \left.+\frac{\mathrm{i}}{16}\left(\gamma_{a}\right)^{\alpha \beta}\left(\left[D_{(\alpha}^{[K}, D_{\beta)}^{L]}\right] \sigma\right) \mathscr{N}_{K L}+\frac{3 \mathrm{i}}{8}\left(\gamma_{a}\right)^{\alpha \beta}\left(D_{(\alpha}^{[K} \sigma\right)\left(D_{\beta)}^{L]} \sigma\right) \mathscr{N}_{K L}\right), \\
S^{I J}= & s^{I J}+2 \mathrm{i}^{2} \frac{\theta^{I J}-s^{K(I} s^{J) L} \theta_{K L}+2 s^{K(I} \theta_{\gamma}^{J)} \theta_{\delta K} x^{\gamma \delta}-\theta^{I J} \Theta_{s}+s^{K(I} \theta^{J)}{ }_{K} \Theta}{1-s^{2} x^{2}-\Theta_{s}+\frac{1}{4} s^{2} \Theta^{2}}
\end{aligned}
$$

where $D_{A}=\left(\partial_{a}, D_{\alpha}^{I}\right)$ are the covariant derivatives of flat $\mathscr{N}$-extended 3D Minkowski superspace.

\section{AdS SUSY and target spaces}

The main question of the papers $[13,20,21]$ was to understand what are the constraints on the target space geometry of a non-linear sigma-model living in the AdS space-time and possessing 
$(p, q)$ supersymmetry. The $(1,0)$ case is trivial. in fact it can be easily proven that any Rimaniann manifold is a good target space for $3 \mathrm{D} \mathscr{N}=1$ AdS supersymmetry. With $\mathscr{N}>1$ there are nontrivial constraints that depend on the $(p, q)$ isometries and the transformations of the matter fields. In the following we will summarize the results of $[13,20,21]$ for the $\mathscr{N}=2,3,4$ cases. Our results are based on the use of the $(p, q)$ AdS superspaces but in the following we will avoid proofs and technical details that the reader can find in the aforementioned papers.

Note that the same question has already been studied in the case of sigma-models in flat 3D Minkowski space-time, see for example [11]. In the flat case, for $\mathscr{N}=2$ the target space is a general Kähler manifold while both for the $\mathscr{N}=3$ and $\mathscr{N}=4$ the target space is a general hyperKähler space. We will see that, depending on the specific case, lifting the sigma-models to AdS impose additional constraints on the target spaces.

\subsection{AdS supersymmetry and target space geometry: $\mathscr{N}=2$}

The $\mathscr{N}=2$ story is pretty simple but still nontrivial and worth to be discussed in details. It was studied in [13]. It is useful to analyze the $\mathscr{N}=2$ geometry by defining complex Grassmannian coordinates $z^{M}=\left(x^{m}, \theta^{\mu}, \bar{\theta}_{\mu}\right), \overline{\theta^{\mu}}=\bar{\theta}^{\mu}$, and covariant derivatives $\left(\mathscr{D}_{a}, \mathscr{D}_{\alpha}, \overline{\mathscr{D}}^{\alpha}\right)$ that closely mimic the well known 4D $\mathscr{N}=1$ covariant derivatives. 3D $\mathscr{N}=2$ off-shell matter multiplets are typically described by chiral superfields. We then consider a set of covariantly chiral superfields $\phi^{\mathrm{a}}, \overline{\mathscr{D}}_{\alpha} \phi^{\mathrm{a}}=$ 0 . Here a $=1, \cdots, d$ with $d$ the target space complex dimension. The general matter model that can be constructed in terms of $\phi^{\text {a }}$ takes the form

$$
S=\int \mathrm{d}^{3} x \mathrm{~d}^{4} \theta E K\left(\phi^{\mathrm{a}}, \bar{\phi}^{\overline{\mathrm{a}}}\right)+\left\{\int \mathrm{d}^{3} x \mathrm{~d}^{2} \theta \mathscr{E} W\left(\phi^{\mathrm{a}}\right)+\text { c.c. }\right\} .
$$

As in the flat case $K$ is the target space Kähler potential, while $W$ is a superpotential. It is worth to explicitly distinguish the specific features of the $(1,1)$ and $(2,0)$ cases.

$(1,1)$ AdS SUSY Given a holomorphic function $F(\phi)$, in the $(1,1)$ AdS case, it holds ${ }^{3}$

$$
\int \mathrm{d}^{3} x \mathrm{~d}^{2} \theta \mathscr{E} F(\varphi)=\int \mathrm{d}^{3} x \mathrm{~d}^{4} \theta E \frac{1}{S} F(\varphi)
$$

The previous equation relates every chiral integral of a holomorphic function to a full superspace integral. It means that a superpotential can always be reabsorbed in the Kähler potential. This also implies that Kähler transformations, $K(\phi, \bar{\phi}) \rightarrow K(\phi, \bar{\phi})+\Lambda(\phi)+\bar{\Lambda}(\bar{\phi})$, are not well defined in $(1,1) \mathrm{AdS}_{3}$ and hence the sigma-model Lagrangian should be globally defined on the target space. As a consequence, the Kähler two-form, $\Omega=2 \mathrm{i} g_{\mathrm{a} \bar{b}} \mathrm{~d} \varphi^{\mathrm{a}} \wedge \mathrm{d} \bar{\varphi}^{\overline{\mathrm{b}}}$, associated with the Kähler metric $g_{\mathrm{a} \overline{\mathrm{b}}}:=\partial_{\mathrm{a}} \partial_{\overline{\mathrm{b}}} K$, is exact and hence the target space is necessarily non-compact. The situation is exactly the same as the $4 \mathrm{D} \mathscr{N}=1 \mathrm{AdS}$ case recently studied in $[17,18,16]$. Then we see that the $\sigma$-model couplings in $(1,1)$ AdS are more restrictive than in the 3D Minkowski case.

For the analysis of the $\mathscr{N}>2$ cases it is crucial to keep in mind that $(1,1)$ AdS supersymmetry necessarily implies the target space to be non-compact.

\footnotetext{
${ }^{3}$ In the complex basis the $(1,1)$ AdS superspace is naturally parametrized by a complex parameter $\mu$ such that $|\mu|=S$. Here we ignore its phase and use $\mu=S$.
} 
$(2,0)$ AdS SUSY In the $(2,0)$ case, in the complex basis, the $S O(2)$ symmetry becomes a $U(1)$ $R$-symmetry phase transformation of the complex covariant derivatives. In general, if the complex superfields $\phi^{\mathrm{a}}$ are charged under the $\mathrm{U}(1)_{R}$, they transformation as $\delta_{\mathrm{U}(1)_{R}} \phi^{\mathrm{a}}=\xi^{\mathrm{a}}(\phi)$. For $R$-invariance, the Kähler potential $K(\phi, \bar{\phi})$ and the superpotential $W(\phi)$ should obey:

$$
\xi^{\mathrm{a}} K_{\mathrm{a}}=\bar{\xi}^{\overline{\mathrm{a}}} K_{\overline{\mathrm{a}}}, \quad \xi^{\mathrm{a}} W_{\mathrm{a}}=-2 W
$$

This implies that $\xi^{\mathrm{a}}$ is a holomorphic Killing vector field on the target space and hence the sigmamodel Kähler manifold possesses an induced U(1) isometry.

On the other hand, if $\delta_{\mathrm{U}(1)_{R}} \phi^{\mathrm{a}}=0$, then the sigma-model target space can be a general Kähler manifold. The price is that in this case the superpotential necessary have to be identically zero.

We conclude by pointing out an important difference between the $(2,0)$ and $(1,1)$ cases. We have observed that, due to eqs. (3.2), the $(1,1)$ sigma-models target spaces need to be non-compact. In the $(2,0)$ case there is no analogue of eq. (3.2), the full and chiral superspace integrals are separated objects and holomorphicity is well defined since it actually holds $\int \mathrm{d}^{3} x \mathrm{~d}^{2} \theta \mathscr{E} F(\varphi)=0$ exactly as in flat superspace. This means that Kähler transformations, $K(\phi, \bar{\phi}) \rightarrow K(\phi, \bar{\phi})+\Lambda(\phi)+$ $\bar{\Lambda}(\bar{\phi})$, leave invariant the sigma-model action and the Kähler potential doesn't need to be globally defined. Then, the $(2,0)$ supersymmetry does not restrict the target space to be compact.

\subsection{Kähler cones}

There is one subclass of $\mathscr{N}=2$ sigma-models that is of particular interests. These are Kähler cones which are the target spaces for general 3D $\mathscr{N}=2$ conformal sigma-models.

A Kähler manifold $\left(\mathscr{M}, g_{\mathrm{a} a}\right)$ parametrized by local complex coordinates $\phi^{\mathrm{a}}$ is called a Kähler cone if it possesses a homothetic conformal Killing vector or infinitesimal dilatation

$$
\chi=\chi^{\mathrm{a}} \frac{\partial}{\partial \phi^{\mathrm{a}}}+\bar{\chi}^{\overline{\mathrm{a}}} \frac{\partial}{\partial \bar{\phi}^{\overline{\mathrm{a}}}} \equiv \chi^{\mu} \frac{\partial}{\partial \varphi^{\mu}},
$$

with the property

$$
\nabla_{v} \chi^{\mu}=\delta_{v}{ }^{\mu} \quad \Longleftrightarrow \quad \nabla_{\mathrm{b}} \chi^{\mathrm{a}}=\delta_{\mathrm{b}}{ }^{\mathrm{a}}, \quad \nabla_{\overline{\mathrm{b}}} \chi^{\mathrm{a}}=\partial_{\overline{\mathrm{b}}} \chi^{\mathrm{a}}=0
$$

In particular, $\chi$ is holomorphic. The properties of $\chi$ include the following:

$$
g_{\mathrm{a} \overline{\mathrm{b}}} \chi^{\mathrm{a}} \bar{\chi}^{\overline{\mathrm{b}}}=K, \quad \chi_{\mathrm{a}}:=g_{\mathrm{a} \overline{\mathrm{b}}} \bar{\chi}^{\overline{\mathrm{b}}}=\partial_{\mathrm{a}} K \quad \Longrightarrow \quad \chi^{\mathrm{a}} K_{\mathrm{a}}=K,
$$

where $K:=g_{\mathrm{a} \overline{\mathrm{b}}} \chi^{\mathrm{a}} \bar{\chi}^{\overline{\mathrm{b}}}$ can be used as a global Kähler potential, $g_{\mathrm{a} \overline{\mathrm{b}}}=\partial_{\mathrm{a}} \partial_{\overline{\mathrm{b}}} K$. Complex coordinates for $\mathscr{M}$ can be chosen such that

$$
\chi=\phi^{\mathrm{a}} \frac{\partial}{\partial \phi^{\mathrm{a}}}+\bar{\phi}^{\overline{\mathrm{a}}} \frac{\partial}{\partial \bar{\phi}^{\overline{\mathrm{a}}}} \quad \longrightarrow \quad \phi^{\mathrm{a}} K_{\mathrm{a}}(\phi, \bar{\phi})=K(\phi, \bar{\phi}) .
$$

We will see that a large class of $\mathscr{N}=3,4$ sigma-models is necessarily a Kähler cone. 


\section{4. $\mathscr{N}=3,4$ AdS supersymmetry and sigma-models target space geometries}

With 3D $\mathscr{N}=3,4$ flat Poincaré supersymmetry the sigma-model target spaces are constrained to be arbitrary hyper-Kähler manifolds; see [11] and references therein. In the 3D $\mathscr{N}=3,4(p, q)$ AdS supersymmetric case, the target spaces are hyper-Kähler manifolds of restricted type [20,21]. Let us make some general quite intuitive observations.

It is clear that every $\mathscr{N}=3,4(p, q)$ AdS sigma-model will automatically be $\mathscr{N}=2$ supersymmetric.

In particular, remember that for $q>0$, the AdS isometry group is $\operatorname{OSp}(p \mid 2 ; \mathbb{R}) \times \operatorname{OSp}(q \mid 2 ; \mathbb{R})$. It is clear that it always exist both a $(1,1) \operatorname{OSp}(1 \mid 2 ; \mathbb{R}) \times \operatorname{OSp}(1 \mid 2 ; \mathbb{R})$ and a $(2,0) \operatorname{OSp}(2 \mid 2 ; \mathbb{R}) \times$ $\mathrm{SL}(2 ; \mathbb{R})$ proper subgroup of the full $(p, q)$ isometries. This fact is equivalent to the observation that every $(p, q)$ AdS superspace with $q>0$ can be reduced to both $(1,1)$ and $(2,0)$ AdS. This simple observation intuitively tells us that for sure the target space geometry will be a non-compact Kähler manifold possessing a $\mathrm{U}(1)$ symmetry. ${ }^{4}$

When $q=0$, the isometry group is a proper subgroup of $\operatorname{OSp}(\mathscr{N} \mid 2 ; \mathbb{R}) \times \operatorname{SL}(2 ; \mathbb{R})$. There is no $(1,1)$ AdS subgroup. This intuitively tells us that in this case it might be possible to have compact target spaces.

Depending on the particular $(p, q)$, the $R$-symmetry group of such supersymmetric sigmamodels includes $\mathrm{SU}(2)$ as a subgroup which induce a $\mathrm{SO}(3)$ symmetry on the target space. It is interesting to note that in $4 \mathrm{D} \mathscr{N}=2$ it was proved that possessing a $\mathrm{SO}(3) R$-symmetry is a sufficient condition for a sigma-model to have as target space a hyper-Kähler cone. The latter are the target spaces of extended superconformal models [11].

To explicitly classify all possible types of hyper-Kähler target space geometries for AdS $\mathscr{N}=$ 3,4 , in [20,21] we developed two different and complementary realizations for the most general $(p, q)$ supersymmetric sigma-models: (i) off-shell formulation in terms of $\mathscr{N}=3,4$ projective supermultiplets; (ii) on-shell formulation based on $(2,0)$ AdS covariantly chiral superfields.

Both approaches are quite technical and we are not going to explain in details their use. But before presenting the results of the classification, we can comment about the general ideas underlying the two approaches.

In the case (i), one uses the off-shell projective superspace formalism developed in [10] for general supergravity-matter couplings applied to the particular $\mathscr{N}=3,4$ AdS backgrounds (see [23] and references therein for review of the projective superspace approach). The main dynamical fields in the projective superspace approach are charged hyper-multiplets described by arctic superfields $\Upsilon^{\mathrm{I}}(z, \zeta)$ which are holomorphic functions of an extra complex variable $\zeta$. The dependence on $\zeta$ implies that $\Upsilon^{\mathrm{I}}(z, \zeta)$ has an infinite number of ordinary auxiliary fields encoded in their Taylor expansion. The superfield $\Upsilon^{\mathrm{I}}(z, \zeta)$ satisfies an analyticity constraint that makes it effectively a function only of half of the full superspace coordinates [10]. It can also be proven that the projective superfields can be consistently reduced to $(2,0)$ superfields for all $\mathscr{N}=3,4(p, q) \operatorname{AdS}[20,21]$. In fact, one can write the general $\mathscr{N}=3,4$ AdS sigma-model action as

$$
S=\oint_{\gamma} \frac{\mathrm{d} \zeta}{2 \pi \mathrm{i} \zeta} \int \mathrm{d}^{3} x \mathrm{~d}^{4} \theta E L\left(\Upsilon^{\mathrm{I}}(\zeta), \breve{\Upsilon}^{\bar{J}}(\zeta)\right)
$$

\footnotetext{
${ }^{4}$ The possibility of having uncharged fields under the $(2,0) \mathrm{U}(1)_{R}$ is not consistent with extra supersymmetry since the sigma-models are based on hyper-multiplets.
} 
where the superspace integration is over the reduced $(2,0)$ superspace and the function $L$ is constrained depending on the type of $(p, q)$ supersymmetry [21]. The beauty of this approach is that by construction the action is fully off-shell supersymmetric. The drawback is that the geometrical datas of the sigma-modes are partially hidden. The way to explicitly read-off such data and compute the hyper-Kähler potential of the sigma-models in principle is to integrate out all the auxiliary superfields in (4.1) by computing the integral over the $\zeta$ variables. By doing so, one remains with an action written only in terms of $(2,0)$ chiral variables and that in general is $\mathscr{N}=3,4$ supersymmetric only on-shell. See [21] for the discussion of this "top-botton" procedure.

In the approach (ii), which is "botton-top", we start directly from a $(2,0)$ sigma-model and then we impose that the following extra supersymmetry transformations

$$
\delta \phi^{\mathrm{a}}=\frac{\mathrm{i}}{2} \overline{\mathscr{D}}^{2}\left(\bar{\rho} \Omega^{\mathrm{a}}(\phi, \bar{\phi})\right),
$$

closes, in general on-shell, and that the $(2,0)$ action is left invariant. Here $\bar{\rho}$ contains the informations about the $(p, q)$ extra supersymmetries [21]. Along this line, the Kähler potential $K(\phi, \bar{\phi})$ and the superfields ${ }^{5} \Omega^{\mathrm{a}}(\phi, \bar{\phi})$ turn out to satisfy a number of constraints that will define the $(p, q)$ target space geometries.

Let us finally list the results for all the possible cases.

\section{$4.1(3,0)$ case}

The $(3,0)$ isometries include an $\mathrm{SO}(3) \cong \mathrm{SU}(2) R$-symmetry group. This property ultimately implies that, for any supersymmetric sigma-model, the $(3,0)$ target space must be a hyper-Kähler cone [20]. Hyper-Kähler cones are the target spaces of $\mathscr{N}=3$ superconformal sigma models [11]. All hyper-Kähler cones are non-compact.

Let us explain in more details what an hyper-Kähler cone is. It simply is a hyper-Kähler manifold $\left(\mathscr{M}, g_{\mu v}, J_{A}{ }^{\mu}{ }_{v}\right)$ admitting an infinitesimal dilatation $\chi$. Here $J_{A}{ }^{\mu}{ }_{v}$ are the three integrable quaternionic complex structures

$$
J_{A} J_{B}=-\delta_{A B} \mathbb{I}+\varepsilon_{A B C} J_{C} .
$$

Associated with the conformal Killing vector field $\chi$ are three Killing vectors $X_{A}{ }^{\mu}:=J_{A}{ }^{\mu}{ }_{v} \chi^{\nu}$, which leave the Kähler potential invariant, $X_{A}{ }^{\mu} \partial_{\mu} K=0$. These obey the SU(2) algebra

$$
\left[X_{A}, X_{B}\right]=-2 \varepsilon_{A B C} X_{C}
$$

Such triplet of Killing vectors is the one ultimately induced by the SU(2) $R$-symmetry transformations of the hyper-multiplets.

\subsection{1 $(2,1)$ case}

In the $(2,1)$ case the $R$-symmetry group is reduced to $\mathrm{SO}(2) \cong \mathrm{U}(1)$. The hyper-multiplets have to be charged under the $\mathrm{U}(1)_{R}$ and this induces a $\mathrm{U}(1)$ isometry on the target space Kähler manifold. Moreover, there is a proper $(1,1)$ supersymmetry, hence the manifold has to be non-compact.

\footnotetext{
${ }^{5}$ The superfield $\Omega^{\mathrm{a}}(\phi, \bar{\phi})$ ultimately generate the quaternionic structures of the hyper-Kähler geometries [21].
} 
Under detailed analysis it turns out that the $(2,1)$ sigma-model target space must be a noncompact hyper-Kähler manifold endowed with a Killing vector field which generates an $\mathrm{SO}(2)$ group of rotations of the two-sphere of quaternionic complex structures. Let us see some properties of such a class of manifolds.

Let $\mathscr{M}$ be a hyper-Kähler manifold equipped with three complex structures $J_{A}{ }^{\mu}{ }_{v}$ along with a $\mathrm{U}(1)$ isometry $V^{\mu}$ which acts as a rotation on them. Without loss of generality, $V^{\mu}$ is holomorphic w.r.t. $J_{3}$

$$
\mathscr{L}_{V} J_{1}=-J_{2}, \quad \mathscr{L}_{V} J_{2}=+J_{1}, \quad \mathscr{L}_{V} J_{3}=0
$$

The three closed Kähler two-forms are

$$
\Omega_{A}=\frac{1}{2}\left(\Omega_{A}\right)_{\mu v} \mathrm{~d} \phi^{\mu} \wedge \mathrm{d} \phi^{v}, \quad\left(\Omega_{A}\right)_{\mu v}=g_{\mu \rho}\left(J_{A}\right)^{\rho}{ }_{v} .
$$

From $\Omega_{1}$ and $\Omega_{2}$ one can construct the complex $(2,0)$ and $(0,2)$ forms with respect to $J_{3}$

$$
\Omega_{ \pm}=\frac{1}{2} \Omega_{1} \pm \frac{\mathrm{i}}{2} \Omega_{2}, \quad \mathscr{L}_{V} \Omega_{ \pm}= \pm \mathrm{i} \Omega_{ \pm} .
$$

Note that $\Omega_{+}$is holomorphic with respect to $J_{3}$. Each of these two-forms is closed by construction. Moreover, due to the properties of the Killing vector $V^{\mu}$, the complex structures $\Omega_{+}$and $\Omega_{-}$prove to be exact. Indeed, if one considers the form $\rho_{+}:=-\mathrm{i} \iota_{V} \Omega_{+}$, which is $(1,0)$ holomorphic with respect to $J_{3}$, it can be seen that $\mathrm{d} \rho_{+}=\Omega_{+}$. Because some of the Kähler two-forms are exact, $\mathscr{M}$ is non-compact [20,21].

It is interesting to note that the target spaces of $(2,1)$ supersymmetric sigma-models in $\mathrm{AdS}_{3}$ are the same as those of $\mathscr{N}=2$ supersymmetric sigma models in $\mathrm{AdS}_{4}$ [16] and $\mathscr{N}=1$ supersymmetric sigma models in $\mathrm{AdS}_{5}$ [15].

\section{$4.2(3,1)$ and $(2,2)$ cases}

In the $\mathscr{N}=4$ case, the conformal supergravity has $\mathrm{SO}(4) \cong\left(\mathrm{SU}(2)_{L} \times \mathrm{SU}(2)_{R}\right) / \mathbb{Z}_{2}$ as $R$ symmetry part of the structure group. The decomposition in left and right $\mathrm{SU}(2)$ groups has a very simple implication on the target space geometry of all the 3D $\mathscr{N}=4$ sigma models. In fact, these target spaces are decomposable

$$
\mathscr{M}_{L} \times \mathscr{M}_{R}
$$

where $\mathscr{M}_{L}$ and $\mathscr{M}_{R}$ are certain hyper-Kähler manifolds. The reason of such decomposition is very simple. Most general $\mathscr{N}=4$ sigma models are described by $\mathscr{N}=4$ hyper-multiplets. There are two independent set of hyper-multiplet charged either under the left or right $S U(2)$ [10]. The same remain true in the $\mathrm{AdS}$ cases even though the $R$-symmetry group is reduced depending on the cases.

The classification of the $(3,1)$ and $(2,2)$ target spaces is exactly the same as in $\mathscr{N}=3$ case. The same arguments apply in these $\mathscr{N}=4$ cases. In particular it holds that [21]:

$(3,1)$ : For any supersymmetric sigma model, its left and right target spaces must be hyper-Kähler cones. This is not surprizing due to the residual SU(2) $R$-symmetry. 
$(2,2)$ : Left and right target spaces must be non-compact hyper-Kähler manifolds possessing a Killing vector field which generates an $\mathrm{SO}(2)$ group of rotations of the two-sphere of complex structures. This is the $\mathscr{N}=4$ analogue of the $(2,1)$ case.

The story is much more interesting in the $(4,0)$ case where we have the deformation parameter $X$ playing a new role.

\section{$4.3(4,0)$ case}

Let us start by writing the $(4,0)$ AdS superspace geometry

$$
\begin{gathered}
\left\{\mathscr{D}_{\alpha}^{I}, \mathscr{D}_{\beta}^{J}\right\}=2 \mathrm{i} \delta^{I J} \mathscr{D}_{\alpha \beta}-4 \mathrm{i} S \delta^{I J} \mathscr{M}_{\alpha \beta}+\mathrm{i} \varepsilon_{\alpha \beta}\left(X \varepsilon^{I J K L} \mathscr{N}_{K L}-4 S \mathscr{N}^{I J}\right), \\
{\left[\mathscr{D}_{a}, \mathscr{D}_{\beta}^{J}\right]=S\left(\gamma_{a}\right)_{\beta}{ }^{\gamma} \mathscr{D}_{\gamma}^{J}, \quad\left[\mathscr{D}_{a}, \mathscr{D}_{b}\right]=-4 S^{2} \mathscr{M}_{a b} .}
\end{gathered}
$$

The constant $X$ is a free parameter that does not affect the bosonic $\operatorname{AdS}_{3}$ body of the $(4,0)$ superspace. The algebra simplifies if we switch from $\mathrm{SO}(4)$ isovector indices, to pairs of $\mathrm{SU}(2)_{L} \times \mathrm{SU}(2)_{R}$ isospinor indices. The $(4,0)$ algebra in isospinor notations takes the form ${ }^{6}$

$$
\begin{aligned}
\left\{\mathscr{D}_{\alpha}^{i \bar{i}}, \mathscr{D}_{\beta}^{j i \bar{j}}\right\} & =2 \mathrm{i} \varepsilon^{i j} \varepsilon^{i \bar{j}} \mathscr{D}_{\alpha \beta}+2 \mathrm{i} \varepsilon_{\alpha \beta} \varepsilon^{i \bar{j}}(2 S+X) \mathbf{L}^{i j}+2 \mathrm{i} \varepsilon_{\alpha \beta} \varepsilon^{i j}(2 S-X) \mathbf{R}^{i \bar{j}}-4 \mathrm{i} S \varepsilon^{i j} \varepsilon^{i \bar{j}} \mathscr{M}_{\alpha \beta}, \\
{\left[\mathscr{D}_{a}, \mathscr{D}_{\beta}^{j \bar{j}}\right] } & =S\left(\gamma_{a}\right)_{\beta} \mathscr{D}_{\gamma}^{j \bar{j}}, \quad\left[\mathscr{D}_{a}, \mathscr{D}_{b}\right]=-4 S^{2} \mathscr{M}_{a b} .
\end{aligned}
$$

It is clear that for general values of $X$ the structure group of the $(4,0)$ algebra is the full $\mathrm{SU}(2)_{L} \times \mathrm{SU}(2)_{R}$. On the other hand, there are two critical cases

$$
X= \pm 2 S
$$

where either $\mathrm{SU}(2)_{L}$ or $\mathrm{SU}(2)_{R}$ disappear from the algebra. Then there are different isometry groups depending on the choice of $X$. This property is lifted to the structure of the left and right target spaces $\mathscr{M}_{L}$ and $\mathscr{M}_{R}$. In fact, depending on the values of $X$ it holds [21]:

$X=0$ : Both the left and right target spaces must be hyper-Kähler cones. This is intuitively associated with the fact that the whole $\mathrm{SU}(2)_{L} \times \mathrm{SU}(2)_{R}$ is preserved. With $X=0$ the sigma-models turns out to be superconformal.

$X \neq \pm 2 S$ : Similarly to the $X=0$ case, its left and right target spaces must be hyper-Kähler cones. The crucial difference when $X \neq 0$ is that the sigma-model is not superconformal. The presence of $X$ leads to non-trivial scalar potentials in both the left and right sectors.

$X= \pm 2 S:$ One of the two target spaces, either left or right, must be a hyper-Kähler cone but not superconformal due to the presence of $X$. On the other hand, the other target space, associated to the sector in which the SU(2) disappears, is an arbitrary hyper-Kähler manifold; in particular, it may be compact.

$S=0$ In the flat case, the presence of a $X \neq 0$ leads to the appearance of nontrivial potentials in both left and right sectors. This implies that the parameter $X$ can be used as a new mechanism to generate massive sigma models in 3D $\mathscr{N}=4$ Minkowski superspace.

\footnotetext{
${ }^{6}$ Here the isospinor indices are $i=1,2, \bar{i}=\overline{1}, \overline{2}$ with the $\mathrm{SU}(2)$ invariants $\varepsilon^{i j}=-\varepsilon^{j i}, \varepsilon^{\bar{i} \bar{j}}=-\varepsilon^{\overline{j i}}$ and $\varepsilon^{12}=\varepsilon^{\overline{1} \overline{2}}=1$. The $\mathrm{SU}(2)_{L}$ and $\mathrm{SU}(2)_{R}$ generators are respectively $\mathbf{L}^{i j}$ and $\mathbf{R}^{\bar{i} \bar{j}}[10]$.
} 


\section{Conclusion}

We have reviewed our recent construction of three-dimensional (3D) $(p, q)$ Anti-de Sitter superspaces and the classification of the target space geometries associated with general supersymmetric $\mathscr{N}=2,3,4$ nonlinear sigma-models in $\mathrm{AdS}_{3}$ based on [10, 13, 20, 21].

From the superspace point of view, it would be interesting to classify more general 3D Lorentzian and Euclidian superspaces admitting various off-shell supersymmetries in analogy to the recent literature on supersymmetry on curved superspaces (see e.g. [18, 24]) In fact, by using general superspace supergravity-matter couplings [20] we then have a formalism to define supersymmetric models in general 3D curved manifolds. This might then find application in studying QFT on such supersymmetric backgrounds along the lines of the localization literature.

Our $(p, q)$ AdS superspaces might also be used to study 3D supersymmetric higher-spin field theories.

\section{Acknowledgments}

I am grateful to my collaborators D. Butter, S. M. Kuzenko and U. Lindström for working with me at various stages of this research. This work was supported by the Australian Research Council's Discovery Early Career Award (DECRA), project No. DE120101498.

\section{References}

[1] J. Bagger and N. Lambert, "Modeling multiple M2's," Phys. Rev. D 75, 045020 (2007); [arXiv:hep-th/0611108]; “Gauge symmetry and supersymmetry of multiple M2-branes," Phys. Rev. D 77, 065008 (2008); [arXiv:0711.0955 [hep-th]]; “Comments on multiple M2-branes,” JHEP 0802, 105 (2008). [arXiv:0712.3738 [hep-th]].

[2] A. Gustavsson, “Algebraic structures on parallel M2-branes,” Nucl. Phys. B 811, 66 (2009). [arXiv:0709.1260 [hep-th]].

[3] O. Aharony, O. Bergman, D. L. Jafferis and J. Maldacena, "N=6 superconformal Chern-Simons-matter theories, M2-branes and their gravity duals," JHEP 0810, 091 (2008). [arXiv:0806.1218 [hep-th]].

[4] W. Li, W. Song and A. Strominger, "Chiral Gravity in Three Dimensions," JHEP 0804, 082 (2008). [arXiv:0801.4566 [hep-th]].

[5] E. A. Bergshoeff, O. Hohm and P. K. Townsend, "Massive Gravity in Three Dimensions," Phys. Rev. Lett. 102, 201301 (2009). [arXiv:0901.1766 [hep-th]].

[6] P. S. Howe and R. W. Tucker, "A locally supersymmetric and reparametrization invariant action for a spinning membrane,” J. Phys. A 10, L155 (1977); “Local supersymmetry in (2+1) dimensions. 1. Supergravity and differential forms," J. Math. Phys. 19, 869 (1978); "Local supersymmetry in (2+1) dimensions. 2. An action for a spinning membrane,” J. Math. Phys. 19, 981 (1978).

[7] M. Brown and S. J. Gates Jr., "Superspace Bianchi identities and the supercovariant derivative," Annals Phys. 122, 443 (1979).

[8] P. S. Howe, J. M. Izquierdo, G. Papadopoulos and P. K. Townsend, "New supergravities with central charges and Killing spinors in 2+1 dimensions,” Nucl. Phys. B 467, 183 (1996). [hep-th/9505032]. 
[9] P. S. Howe and E. Sezgin, “The Supermembrane revisited,” Class. Quant. Grav. 22, 2167 (2005). [hep-th/0412245].

[10] S. M. Kuzenko, U. Lindström and G. Tartaglino-Mazzucchelli, "Off-shell supergravity-matter couplings in three dimensions," JHEP 1103, 120 (2011). [arXiv:1101.4013 [hep-th]].

[11] S. M. Kuzenko, J. -H. Park, G. Tartaglino-Mazzucchelli and R. Unge, “Off-shell superconformal nonlinear sigma-models in three dimensions,” JHEP 1101, 146 (2011). [arXiv:1011.5727 [hep-th]].

[12] A. Achúcarro and P. K. Townsend, "A Chern-Simons action for three-dimensional anti-de Sitter supergravity theories,” Phys. Lett. B 180, 89 (1986).

[13] S. M. Kuzenko and G. Tartaglino-Mazzucchelli, "Three-dimensional N=2 (AdS) supergravity and associated supercurrents," JHEP 1112, 052 (2011). [arXiv:1109.0496 [hep-th]].

[14] D. Butter and S. M. Kuzenko, “N=2 AdS supergravity and supercurrents," JHEP 1107, 081 (2011) [arXiv:1104.2153 [hep-th]].

[15] J. Bagger and C. Xiong, “Ad $S_{5}$ Supersymmetry in N=1 Superspace,” JHEP 1107, 119 (2011). [arXiv:1105.4852 [hep-th]].

[16] D. Butter and S. M. Kuzenko, “N=2 supersymmetric sigma-models in AdS,” Phys. Lett. B 703, 620 (2011); [arXiv:1105.3111 [hep-th]]; "The structure of $\mathrm{N}=2$ supersymmetric nonlinear sigma models in AdS4," JHEP 1111, 080 (2011). [arXiv:1108.5290 [hep-th]].

[17] A. Adams, H. Jockers, V. Kumar and J. M. Lapan, "N=1 sigma models in $A d S_{4}$," JHEP 1112, 042 (2011). arXiv:1104.3155 [hep-th].

[18] G. Festuccia and N. Seiberg, "Rigid supersymmetric theories in curved superspace," JHEP 1106, 114 (2011). [arXiv:1105.0689 [hep-th]].

[19] V. Pestun, "Localization of gauge theory on a four-sphere and supersymmetric Wilson loops," [arXiv:0712.2824 [hep-th]]; "Localization of the four-dimensional N=4 SYM to a two-sphere and 1/8 BPS Wilson loops," [arXiv:0906.0638 [hep-th]]; D. L. Jafferis, “The exact superconformal R-symmetry extremizes Z," arXiv:1012.3210 [hep-th].

[20] S. M. Kuzenko, U. Lindstrom and G. Tartaglino-Mazzucchelli, "Three-dimensional (p,q) AdS superspaces and matter couplings," JHEP 1208, 024 (2012). [arXiv:1205.4622 [hep-th]].

[21] D. Butter, S. M. Kuzenko and G. Tartaglino-Mazzucchelli, "Nonlinear sigma models with AdS supersymmetry in three dimensions," JHEP 1302, 121 (2013). [arXiv:1210.5906 [hep-th]].

[22] I. A. Bandos, E. Ivanov, J. Lukierski and D. Sorokin, "On the superconformal flatness of AdS superspaces,” JHEP 0206, 040 (2002), [hep-th/0205104].

[23] U. Lindstrom and M. Rocek, "Properties of hyperkahler manifolds and their twistor spaces," Commun. Math. Phys. 293, 257 (2010). [arXiv:0807.1366 [hep-th]]; S. M. Kuzenko, "Lectures on nonlinear sigma-models in projective superspace,” J. Phys. A 43, 443001 (2010). [arXiv:1004.0880 [hep-th]].

[24] C. Klare, A. Tomasiello and A. Zaffaroni, "Supersymmetry on Curved Spaces and Holography," JHEP 1208, 061 (2012). [arXiv:1205.1062 [hep-th]]; D. Cassani, C. Klare, D. Martelli, A. Tomasiello and A. Zaffaroni, "Supersymmetry in Lorentzian Curved Spaces and Holography," [arXiv:1207.2181 [hep-th]]. 